\title{
Macrophage activation syndrome in a child with systemic onset of juvenile idiopathic arthritis
}

\author{
BARBARA STANIMIROVIĆ ${ }^{1}$ STOJISLAV KONJEVIĆ ${ }^{1}$ BILJANA ĐURĐEVIĆ-BANJAC ${ }^{1}$, DARIO ĐUKIĆ \\ ${ }^{1}$ Clinic of Paediatrics, University Hospital Clinical Centre, Banja Luka, Bosnia and Herzegovina \\ ${ }^{2}$ General Hospital Gradiška, Gradiška, Bosnia and Herzegovina, \\ ${ }^{3}$ Clinic of Internal Medicine, University Hospital, Clinical Centre, Banja Luka, Bosnia and Herzegovina
}

Corresponding author:

Barbara Stanimirović

Clinic of Paediatrics

University Hospital, Clinical Centre

12 Beba bb, 78000 Banja Luka

Bosnia and Herzegovina

Phone: 0038751342280

Fax: 0038751342463

E-mail: barbarastanimirovic@gmail.com

\section{ABSTRACT}

Macrophage activation syndrome (MAS) is a serious and potentially fatal complication of various diseases, mainly systemic inflammatory connective tissue disorders, the most frequent being systemic juvenile idiopathic arthritis (sJIA). Its most important clinical and laboratory manifestations are non-remitting fever, hepatosplenomegaly, impaired liver function, cytopenia, hypoalbuminemia, hypertriglyceridemia, hypofibrinogenemia and hyperferritinemia. The prognosis for the syndrome is often poor, so a prompt diagnosis and the appropriate medical treatment are critical for survival. We report a case of an 8-year-old boy who developed MAS as a complication of sJIA. The boy presented with liver insufficiency, impaired coagulation, encephalopathy and respiratory failure and was treated in intensive care unit (ICU). He has achieved complete remission of the disease after therapy with pulse doses of corticosteroids and ciclosporin.

Key words: macrophage activation syndrome, rheumatic diseases, haemophagocytic lymphohistiocytosis

\section{INTRODUCTION}

MAS is a serious, potentially fatal complication of rheumatic diseases, which is seen most frequently in systemic juvenile idio- pathic arthritis (SJIA) and in its adult equivalent, adult-onset Still's disease, although it is increasingly reported in other paediatric inflammatory disorders, namely juvenile systemic lupus erythematosus and Kawasaki disease. $(1,2)$ In recent years, this condition has also been observed in periodic fever syndromes. MAS may occur spontaneously, as a complication of active underlying disease, or may be triggered by an infection, a change in drug therapy or a toxic effect of a medication, including biologics. (1) Clinically, patients with MAS present with non-remitting high fever, pancytopenia, hepatosplenomegaly, hepatic dysfunction, encephalopathy, coagulation abnormalities and sharply increased levels of ferritin. The pathognomonic feature of the syndrome is seen on bone marrow examination, which reveals numerous morphologically benign macrophages exhibiting haemophagocytic activity (figure 1). Such cells may also be found in lymph nodes and spleen, but they may infiltrate almost any organ in the body and may account for many of the systemic features of the syndrome. As MAS bears a close resemblance to a group of histiocytic disorders collectively known as haemophagocytic lymphohistiocytosis (HLH), it is currently classified among the secondary, or acquired, forms of HLH (3)

\section{CASE REPORT}

The patient is an 8-year-old boy diagnosed with sJIA in December 2012. He was treated by a paediatrician in a regional hospital with low doses of prednisone and methotrexate (MTX) with partially controlled disease. In August 2013 he presented with daily (quotidian) fever, pain and swelling of both knees and the left wrist with evanescent, salmon-colouredrash. Laboratory analyses have shown increased parameters of inflammation, anaemia of chronic disease, and thrombocytosis with high ferritin level. In September 2014 he suddenly presented with mucocutaneous bleeding, seizures and breathing difficulties and was admitted to our hospital. The boy was unconscious during examination (Glasgow coma score 4), hypotensive, had high grade fever, hemorrhagic skin rash, mucosal bleeding, tachycardia, tachypnea, hepatosplenomegaly and axillar lymphadenopathy. He had repeating tonic-clonic seizures.

On examination the patient had anemia ( $\mathrm{Hb}$ $8.2 \mathrm{~g} / \mathrm{dL})$, trombocytopenia $\left(26 \times 10^{3} / \mathrm{L}\right)$, elevated serum lactate dehydrogenase (1670 U/L) and liver enzyme levels (aspartate aminotransferase $268 \mathrm{U} / \mathrm{L}$ and alanine aminotransferase $356 \mathrm{U} / \mathrm{L}$ ), prolonged prothrombin time $(1,9)$, reduced serum levels of fibrinogen $(78 \mathrm{mg} / \mathrm{dL})$, increased triglycerides (455 mg/dL) and ferritin (4600 ng/ $\mathrm{mL})$.

He was admitted to the ICU, intubated, put on ventilatory support, received parenteral fluid replacement and inotropic agent. $\mathrm{He}$ received transfusion of fresh frozen plasma and thrombocyte concentrate that con- 
trolled the bleeding. Convulsions were stopped with midazolam. Bone marrow examination revealed haemophagocytosis (figure 1). Lumbar punction was in reference values. He was diagnosed with MAS and treated with 5 pulses of methylprednisolone $30 \mathrm{mg} / \mathrm{kg} /$ day, followed by oral prednisone (PDN) $2 \mathrm{mg} / \mathrm{kg} /$ day and ciclosporin (CSA) $5 \mathrm{mg} / \mathrm{kg} /$ day. MTX was suspended.

The disease remitted after eight weeks. His last laboratory results showed the normalization of ferritin $(190 \mathrm{ng} / \mathrm{mL})$, triglycerides $(64 \mathrm{mg} / \mathrm{dL})$, haemoglobin $(12.7 \mathrm{~g} / \mathrm{dL})$, fibrinogen $(240 \mathrm{mg} / \mathrm{mL})$, AST $(23 \mathrm{U} / \mathrm{L})$ and ALT (30 U/L). The patient is currently treated with PDN $5 \mathrm{mg} /$ day and CSA 6 $\mathrm{mg} / \mathrm{kg} /$ day.

\section{DISCUSSION}

MAS is a life-threatening condition associated with high mortality rates. Although the reported mortality rates for MAS reach $20 \%$, the clinical presentation of macrophage activation syndrome (MAS) is generally acute and occasionally dramatic. (1 - 3) Typically, patients become acutely ill with the sudden onset of non-remitting high fever, profound depression in all 3 blood cell lines (i.e. leukopenia, anaemia, and thrombocytopenia), hepatosplenomegaly, lymphadenopathy, and elevated serum liver enzyme levels. MAS is overt in $10 \%$ of children with sJIA, but occurs subclinically in another $30-40 \%$. It is important to emphasize that the diagnosis of MAS is often a challenge as it may mimic a flare of the underlying disease. Therefore, it is difficult to distinguish sJIA disease flare from MAS. There are no validated criteria for the diagnosis of MAS. A 2014 retrospective analysis concluded that HLH-2004 guidelines are likely not appropriate for identification of MAS in children with sJIA. Ravelli et al. proposed diagnostic guidelines for MAS complicating sJIA, based on expert consensus. These preliminary MAS guidelines showed the strongest ability to identify MAS in sJIA. The diagnosis of macrophage activation syndrome requires the presence of any 2 or more of the following laboratory criteria or 2 or more of the following clinical criteria (table 1). (4)

The treatment for MAS is based on corticosteroids and CSA. CSA has been proven as effective therapy in patients with severe disease and corticosteroid resistant. (5 - 7) That is why it was introduced early in the treatment.

MAS can be triggered by an infection, a change in drug therapy or a toxic effect of a medication, including biologics. (8) We consider that the trigger for MAS in the case of our patient was the inadequate treatment by the paediatrician in the regional hospital and unsatisfactory disease monitoring.

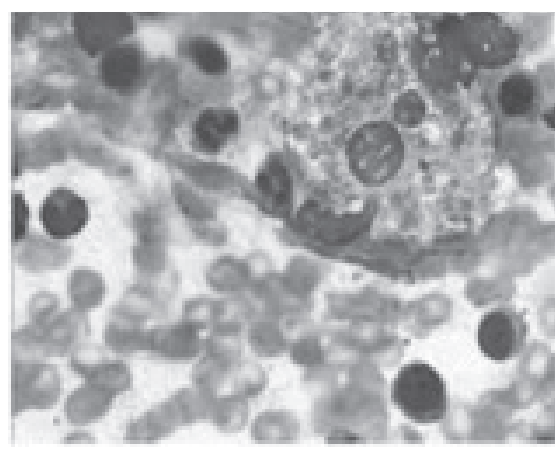

Figure 1: bone marrow aspirate showing macrophage with engulfed red blood cells
Table 1. Preliminary diagnostic guidelines for macrophage activation syndrome complicating systemic juvenile idiopathic arthritis

\begin{tabular}{|c|}
\hline LABORATORY CRITERIA \\
\hline o Decreased platelet count $(<262 \times 109 / \mathrm{L})$ \\
\hline $\begin{array}{l}\text { o Elevated aspartate aminotransferase levels } \\
(>59 \mathrm{U} / \mathrm{L})\end{array}$ \\
\hline o Decreased WBC count $(<4 \times 109 / \mathrm{L})$ \\
\hline o Hypofibrinogenemia $(\leq 2.5 \mathrm{~g} / \mathrm{L})$ \\
\hline CLINICAL CRITERIA \\
\hline $\begin{array}{l}\text { o CNS dysfunction (e.g. irritability, disorien- } \\
\text { tation, lethargy, headache, seizures, coma) }\end{array}$ \\
\hline $\begin{array}{l}\text { o Hemorrhages (e.g. purpura, easy bruising, } \\
\text { mucosal bleeding) }\end{array}$ \\
\hline $\begin{array}{l}\text { o Hepatomegaly ( } \geq 3 \mathrm{~cm} \text { below the costal } \\
\text { margin) }\end{array}$ \\
\hline HISTOPATHOLOGIC CRITERION \\
\hline
\end{tabular}

- Evidence of macrophage hemophagocytosis is found in the bone marrow aspirate sample. The demonstration of hemophagocytosis in bone marrow samples may be required in doubtful cases.

CNS - central nervous system, WBC white blood cell count

\section{CONCLUSION}

MAS is a serious complication of systemic rheumatic disorders, primarily sJIA, associated with long-term morbidity and mortality. The purpose of this case report is to motivate paediatricians for early recognition of the clinical manifestations of MAS. Early diagnosis and aggressive therapeutic approach are the critical points in MAS management and the ones capable of modifying patient's outcome.

\section{REFERENCES}

1. Cassidy JT, Petty RE, Laxer RM, Lindsley CB. Textbook of pediatric rheumatology: macrophage activation syndrome,45, 6th ed. Elsevier Saunders; 2011. p. 674-81.

2. Sawhney S, Woo P, Murray KJ. Macrophage activation syndrome: a potentially fatal complication of rheumatic disorders. Arch Dis Child 2001;85:421-6.

3. Ravelli A. Macrophage activation syndrome. Curr Opin Rheumatol. 2002;14:548-52.

4. Ravelli A, Magni-Manzoni A, Pistorio A, Besana C, Foti T,Ruperto N, et al. Preliminary diagnostic guidelines for macrophage activation syndrome complicating systemic juvenile idiopathic arthritis. J Pediatr. 2005;146:598-604.

5. Emmenegger U, Reimers A, Frey U, et al. Reactive macrophage activation syndrome: a simple screening strategy and its potential in early treatment initiation. Swiss Med Wkly 2002; 132: 230-236.

6. Weitzman S. Approach to hemophagocytic syndromes. AmSoc Hematol. 2011;1:178-83.9

7. Stephan JL, Kone-Paut I, Galambrun C, Mouy R, Bader-Meunier B, Prieur AM. Reactive haemophagocytic syndrome in children 
with inflammatory disorders. A retrospective study of 24 patients. Rheumatology 2001; 40: 1285-1292.

8. Ravelli A, Caria MC, Buratti S, Mulattia C, Temporini F, MartiniA. Methotrexate as a possible trigger of macrophage activation syndrome in systemic juvenile idiopathic arthritis. J Rheumatol. 2001;28:865-7 